\title{
Photonic Hypercrystals
}

\author{
Evgenii E. Narimanov \\ School of Electrical and Computer Engineering and Birck Nanotechnology Center, Purdue University, \\ West Lafayette, Indiana 47907, USA \\ (Received 24 February 2014; revised manuscript received 9 July 2014; published 27 October 2014)
}

\begin{abstract}
We introduce a new "universality class" of artificial optical media-photonic hypercrystals. These hyperbolic metamaterials, with periodic spatial variation of dielectric permittivity on subwavelength scale, combine the features of optical metamaterials and photonic crystals. In particular, surface waves supported by a hypercrystal possess the properties of both the optical Tamm states in photonic crystals and surface-plasmon polaritons at the metal-dielectric interface.
\end{abstract}

DOI: 10.1103/PhysRevX.4.041014

\section{INTRODUCTION}

Metamaterials [1] and photonic crystals [2] currently represent the primary building blocks for novel nanophotonic devices. With the goal of ultimate control over the light propagation, an artificial optical material must rely on either the effect of a subwavelength pattern that changes the average electromagnetic response of the medium [3,4] or on Bragg scattering of light due to a periodic spatial variation that is comparable to the wavelength $[5,6]$. By virtue of this inherent scale separation, the corresponding metamaterial and photonic crystal concepts are generally considered mutually exclusive within the same environment.

The situation is, however, dramatically different in the world of hyperbolic metamaterials [7-9], where the opposite signs of the dielectric permittivity components in two orthogonal directions $\left(\epsilon_{n} \epsilon_{\tau}<0\right)$ lead to the hyperbolic dispersion of TM-polarized propagating waves,

$$
k_{\tau}^{2} / \epsilon_{n}+k_{n}^{2} / \epsilon_{\tau}=\omega^{2} / c^{2},
$$

with the wave numbers unlimited by the frequency $\omega$. As a result, a periodic variation in the dielectric permittivity, regardless of how small its period $d$ is (Fig. 1), will necessarily cause Bragg scattering of these high- $k$ waves, leading to the formation of photonic band gaps in both the wave number and the frequency domains-see Fig. 2. Interestingly, while photonic crystals formed by hyperbolic media have been considered earlier [10,11], with the emphasis on omnidirectional band gaps [10] and GoosHänchen shift [11], these studies generally focused on the photonic crystal regime $d \sim \lambda_{0}$ and thus avoided the

Published by the American Physical Society under the terms of the Creative Commons Attribution 3.0 License. Further distribution of this work must maintain attribution to the author(s) and the published article's title, journal citation, and DOI.
Subject Areas: Metamaterials, Photonics, Plasmonics

hypercrystal limit $d \ll \lambda_{0}$, where $\lambda_{0}$ is the corresponding free-space wavelength.

This effect on the wave propagation and dispersion by phase-space band-gap formation, in what is essentially the metamaterial limit, allows for an unprecedented degree of control of light propagation in photonic hypercrystals. Furthermore, hypercrystals allow one to substantially reduce the highly detrimental effect of the material loss in plasmonic devices and systems. While it was realized that coupling of photons to charges at metal interfaces allows subdiffraction-limit localization of light that has revived the field of surface plasmons [12], the subwavelength confinement is also the regime of the highest

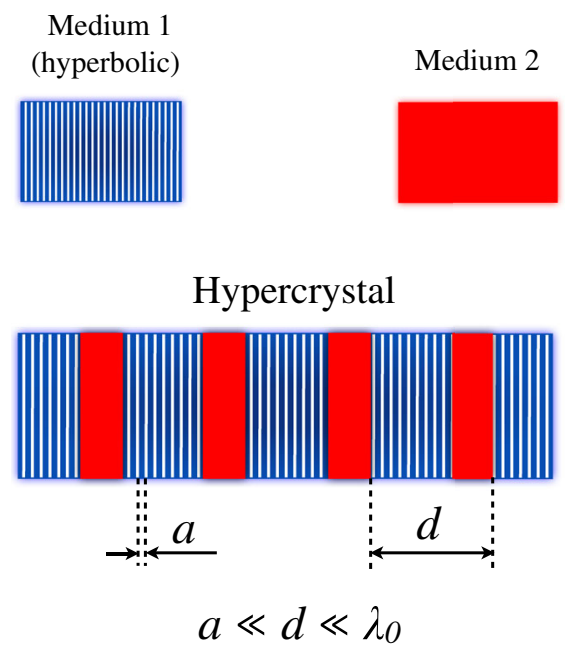

FIG. 1. A hypercrystal is formed by introducing a periodic variation in a hyperbolic medium, with the period smaller than the free-space wavelength $d \ll \lambda_{0}$ but well above the unit cell size of the hyperbolic (meta)material: $d \gg a$. The desired periodic variation can be achieved by introducing a second medium (which could be either a metal, or a dielectric, or another hyperbolic medium with a different dielectric permittivity tensor) in the design of the composite. 


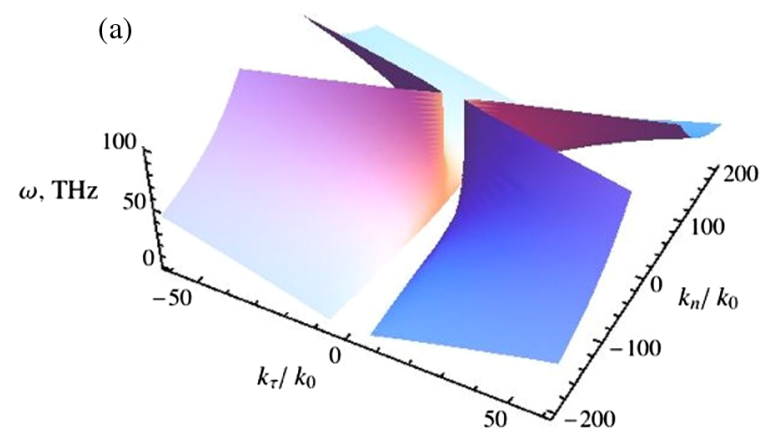

(b)

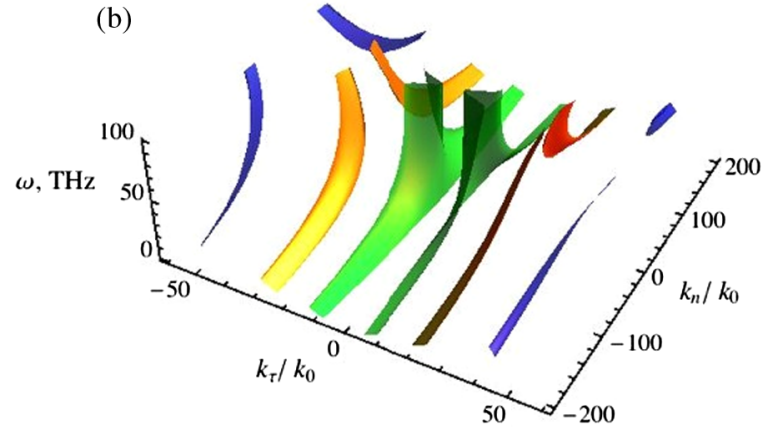

FIG. 2. The comparison of the effective medium dispersion of a photonic hypercrystal (a) to the exact solution (b) in the lossless limit. The hypercrystal unit cell is formed by $250 \mathrm{~nm}$ of semiconductor hyperbolic metamaterial introduced in Ref. [9] (25\% $n^{+}$-doped $\mathrm{In}_{0.53} \mathrm{Ga}_{0.47} \mathrm{As}$ with a $5 \mu \mathrm{m}$ plasma wavelength and $75 \% \mathrm{Al}_{0.48} \mathrm{In}_{0.52} \mathrm{As}$ ), followed by a $250-\mathrm{nm}$ dielectric layer of $\mathrm{Al}_{0.48} \mathrm{In}_{0.52}$ As.

surface-plasmon propagation loss-as it is the photonelectron coupling which is the origin of both of these effects.

Although there is an alternative mechanism of the optical-surface-state formation due to Bragg reflection in the band gap of a photonic crystal that is free from high loss, responsible for the so-called optical Tamm [13-16] or Shockley [17,18] states [19], it normally does not lead to a subwavelength localization. However, in a photonic hypercrystal where Bragg reflections and associated band gaps persist into the metamaterial limit (see Fig. 2), the Tamm mechanism is no longer subject to such limitations. Now, it can lead to the formation of electromagnetic surface states with high wave numbers that are localized at the scale that is much shorter than the free-space wavelength. Furthermore, with Bragg reflections taking part of the "load" in light confinement, when compared to the conventional surface-plasmon polaritons at the metal-dielectric interface, these "hyperplasmons" can show both stronger localization (larger wave numbers) and lower loss.

\section{PHOTONIC HYPERCRYSTALS: THE CONCEPT}

Depending on the relation between the optical wavelength $\lambda_{0}$ and its unit cell size $a$, an artificial composite material generally shows two distinct and qualitatively different regimes for wave propagation and scattering. In the metamaterial limit $a \ll \lambda_{0}$ [1], the electromagnetic response of the composite can be described in terms of its effective permittivity and permeability tensors, whose elements are defined by the geometry and the composition of the unit cell [3]. In contrast to this behavior, optical Bragg scattering in the photonic crystal regime $a \gtrsim \lambda_{0}$ leads to a nontrivial wave dispersion that can no longer be described by the averaged refractive index; it also leads to the formation of the band gaps in the propagating wave spectrum [2].

However, this separation into two distinct regimes tacitly assumes that the wave numbers of the propagating modes supported by the composite are within the same order of magnitude as the corresponding free-space value. Indeed, in such case the strong inequality $a \ll \lambda_{0}$ implies that the phase $k a$ accumulated by the propagating wave across a single cell of a metamaterial is much smaller than unity, and the effective medium description of the composite is well justified [20].

While generally appropriate for most optical materials, this assumption is violated in hyperbolic media where the dielectric permittivities in two orthogonal directions (e.g., $\epsilon_{x}=\epsilon_{y} \equiv \epsilon_{\tau}$ and $\epsilon_{z} \equiv \epsilon_{n}$ ) have opposite signs, leading to the hyperbolic dispersion of TM-polarized propagating waves

$$
k_{\tau}^{2}-\left(-\frac{\epsilon_{\tau}}{\epsilon_{n}}\right) k_{n}^{2}=\epsilon_{\tau} \frac{\omega^{2}}{c^{2}}
$$

as shown in Fig. 2(a) for the case of semiconductor hyperbolic metamaterials based on an $\operatorname{In}_{0.53} \mathrm{Ga}_{0.47} \mathrm{As}$ : $\mathrm{Al}_{0.48} \mathrm{In}_{0.52}$ As superlattice [9]. Note that the optical hyperbolic media can be found in both "artificial" structures (based, e.g., on doped semiconductors [9], metals [21], and polar constituents [22]) and in the "natural" form (e.g., sapphire [23], bismuth [24-27], triglycine sulfate (TGS) [28-30], and graphite [31]). With the validity of Eq. (2) only limited by the unit cell size of the medium $a$, a hyperbolic metamaterial generally supports a broad spectrum of high- $k$ waves, leading to the super-singularity in the photonic density of states [32] and a wide range of related phenomena-from quantum-electrodynamic effects [3335 ] to enhanced scattering and reduced reflectivity [36].

As a result, a periodic variation introduced in a hyperbolic medium, even with the period $d \ll \lambda_{0}$, will lead to strong Bragg scattering of the high- $k$ propagating wavesdespite the fact that the composite remains within the formal bounds of the "metamaterial limit." Note that such behavior is not observed in either conventional metamaterials or in regular photonic crystals. Furthermore, as opposed to the case of a conventional photonic crystal, the resulting wave dispersion in hypercrystals shows a substantially more complex "phase diagram," with multiple 
allowed and forbidden bands in both frequency and momentum dimensions (see Fig. 2).

Formally, we define the photonic hypercrystal regime as the limit

$$
a \ll d \ll \lambda_{0}
$$

for a photonic crystal, with the period $d$ formed by a (either natural or artificial) hyperbolic medium with the unit cell size $a$ (see Fig. 1). With the unit cell size for existing hyperbolic metamaterials on a scale that ranges from about $10 \mathrm{~nm}$ at visible frequencies $[21,37,38]$ to about $100 \mathrm{~nm}$ in mid-IR [9], the ratio $\lambda_{0} / a \sim 100$ allows for a sufficient "separation of scales" to satisfy Eq. (3). Furthermore, with the use of natural hyperbolic materials such as sapphire [23] or bismuth [27], Eq. (3) reduces to simply

$$
d \ll \lambda_{0} \text {. }
$$

\section{A. Hypercrystal in the lossless limit}

To illustrate the new possibilities brought about by the hypercrystals, compared to regular metamaterials and photonic crystals, we first consider the limit in which the effects of the material loss can be neglected. Using the example of a semiconductor hyperbolic metamaterial based on an $\mathrm{In}_{0.53} \mathrm{Ga}_{0.47} \mathrm{As}: \mathrm{Al}_{0.48} \mathrm{In}_{0.52} \mathrm{As}$ superlattice from Ref. [9] and setting the loss to zero, for the "metallic" (permittivity $\epsilon_{m}$ ) and the "dielectric" (permittivity $\epsilon_{d}$ ) components of this hyperbolic metamaterial in the midIR frequency range, we find

$$
\begin{aligned}
& \epsilon_{m}=12.15\left(1-\frac{\omega_{p}^{2}}{\omega^{2}}\right), \\
& \epsilon_{d}=10.23,
\end{aligned}
$$

with the corresponding dielectric permittivity tensor for the metamaterial formed by the layers of these media given by

$$
\begin{aligned}
& \epsilon_{\tau}=p \epsilon_{m}+(1-p) \epsilon_{d}, \\
& \epsilon_{n}=\frac{1}{\frac{p}{\epsilon_{m}}+\frac{1-p}{\epsilon_{d}}},
\end{aligned}
$$

where $p$ is the volume fraction of the metallic phase, and the plasma frequency $\omega_{p}$ can be adjusted within the mid-IR range by the level of doping. Note that the use of the effective medium theory in Eqs. (7) and (8), corresponding to the quasistatic approximation $k a \ll 1$ [39], limits the maximum wave number that can be described by this model to $k_{\max } \sim 1 / a$.

For the unit cell of the idealized hypercrystal of the present section, we take a 250-nm-wide layer of such hyperbolic media with $p=0.25$ and the plasma wavelength of $\lambda_{p} \equiv 2 \pi c / \omega_{p}=5 \mu \mathrm{m}$ (showing the hyperbolic response for all frequencies below $\omega_{p} \approx 377 \mathrm{ps}^{-1}$ ), followed by $250 \mathrm{~nm}$ of the dielectric $\mathrm{Al}_{0.48} \mathrm{In}_{0.52} \mathrm{As}$.

If applied to the hypercrystal, the effective medium approximation would return the permittivity tensor with the dielectric behavior $\left(\epsilon_{\tau}^{\text {eff }}, \epsilon_{n}^{\text {eff }}>0\right)$ for $230 \mathrm{ps}^{-1} \lesssim \omega \lesssim$ $316 \mathrm{ps}^{-1}$ and $\omega \gtrsim 377 \mathrm{ps}^{-1}$, and the hyperbolic response at $316 \mathrm{ps}^{-1} \lesssim \omega \lesssim 377 \mathrm{ps}^{-1}$ (with $\epsilon_{\tau}^{\text {eff }}>0, \epsilon_{n}^{\text {eff }}<0$ ) and $\omega \lesssim 230 \mathrm{ps}^{-1}$ (with $\epsilon_{\tau}^{\text {eff }}<0, \epsilon_{n}^{\text {eff }}>0$ ). For the latter band, this is illustrated in Fig. 2(a), where we plot the frequency as a function of both in-plane and normal to the layer components of the propagating wave momentum. Note that a cross section of this surface in Fig. 2(a) at fixed $\omega$ is indeed a hyperbola.

The effective medium approach, however, does not adequately describe the propagating waves in the hypercrystal-as seen from Fig. 2(b), which shows the exact solution for this model system. Indeed, as expected from the qualitative discussion of the previous section, instead of a single surface [together with its mirror image - see Fig. 2(a)] we find multiple allowed and prohibited bands in both the frequency and momentum dimensions.

The formation of these high- $k$ sub-bands represents the primary difference of the hypercrystal from the regular photonic crystals. This behavior can be understood, e.g., in terms of an effective tight-binding model $[2,40]$, where narrow hyperbolic waveguides that support high- $k$ modes [41] are "coupled" by either metallic or dielectric barrierswith different high- $k$ bands evolving from each of the high- $k$ modes of those waveguides.

While such multiple higher-order band gaps in both frequency and momentum are clearly seen in the dispersion diagram of Fig. 2(b), note that they are only observed for $k_{\tau}>k_{0}$ and therefore are not accessible for light incident from air on a defect-free surface of the hypercrystal. However, many optical phenomena-from light scattering [36] to near-field radiative thermal transport [42-46] to coherent thermal radiation [47] to spontaneous emission [33] - are strongly affected by the high- $k$ part of the wave number spectrum in the system.

\section{B. "Real-world" hypercrystals}

The actual practical realizations of the concept of a hypercrystal are subject to a number of limitations. First, the finite size of the unit cell forming the hyperbolic "phase" of the composite sets the maximum accessible wave number at $k_{\max } \sim 1 / a$ and thus limits the total number of propagating and forbidden bands. Unless a natural hyperbolic medium is available for the desired frequency, even for planar fabrication which so far offers the smallest controllable size of the unit cell, lowering $a$ to the scale of about $10 \mathrm{~nm}$ is usually at the cost of a substantial increase of the electron surface scattering and associated loss. Furthermore, with semiconductor superlattice metamaterials [9], the reduction of $a$ below $50 \mathrm{~nm}$ is only 
accomplished at the expense of lowering the plasma frequency, as electron quantization in sub-50-nm layers limits the maximum doping that can be accommodated before the onset of above-barrier electron transport normal to the layers.

The consequences of the material loss are even more severe. Absorption that is generally inevitable in hyperbolic media eliminates the clear-cut distinction between the forbidden and propagating bands in the phase space of the hypercrystal seen in the lossless limit [as, e.g., in Fig. 2(b)]. Instead, for any frequency $\omega$ and in-plane momentum $k_{\tau}$, a wave in a hypercrystal shows exponential decay, with the corresponding extinction coefficient originating primarily from absorption in the formerly propagating bands and mostly from Bragg reflection in the originally forbidden parts of the phase space. However, a more careful study of the propagating modes of an absorbing photonic hypercrystal still uncovers the signatures of the phase-space structure of the loss-free system.

To describe the phase space of a lossy hypercrystal with axial symmetry (such as, e.g., a layered composite), we introduce the dimensionless "extinction coefficient"

$$
\alpha \equiv \operatorname{Im}\left[k_{n}\right] d,
$$

where $k_{n}$ is the wave-vector component along the optical axis of the composite $z$, so the intensity decays as $I(z) \propto \exp (-2 \alpha z / d)$. In a propagating band, the extinction coefficient is relatively small and entirely defined by the material absorption, with $\alpha=0$ in the lossless limit. In contrast, within a band gap, $\alpha=\mathcal{O}(1)$. The propagating band's "valleys" and band-gap "ridges" in a 3D plot of the extinction coefficient vs the wave number $k_{\tau}$ and frequency $\omega$ thus allow a straightforward visualization of the phase space of a hypercrystal.

We now use this approach for a semiconductor superlattice hypercrystal. In this example, the single period of the composite is defined by a 100-nm layer of an $n^{+}$-doped semiconductor $\operatorname{In}_{0.53} \mathrm{Ga}_{0.47}$ As with the plasma wavelength $\lambda_{p}=5 \mu \mathrm{m}$, followed by a 400-nm-thick semiconductor hyperbolic metamaterial-see Fig. 3. Taking into account the material loss in such heavily doped $\mathrm{In}_{0.53} \mathrm{Ga}_{0.47} \mathrm{As}$, the dielectric permittivity of this semiconductor can be expressed as [9]

$$
\epsilon=12.15\left(1-\frac{\omega_{p}^{2}}{\omega^{2}+i \omega / \tau}\right)
$$

with the relaxation time $\tau \approx 0.16 \mathrm{ps}$ and the plasma frequency $\omega_{p}=2 \pi c / \lambda_{p}$.

In this example (see Fig. 4), the unit cell of the hyperbolic metamaterial consists of interleaving 50-nm-wide layers of the dielectric $\mathrm{Al}_{0.48} \mathrm{In}_{0.52}$ As [with the permittivity in the mid-IR range approximately equal to 10.23 [9]—see also Eq. (6)] and an $n^{+}$-doped $\operatorname{In}_{0.53} \mathrm{Ga}_{0.47}$ As semiconductor

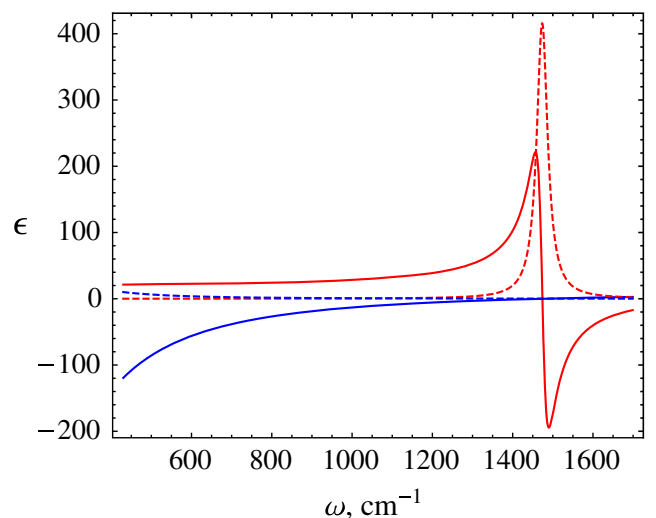

FIG. 3. The dielectric permittivity tensor of an $n^{+}-\mathrm{In}_{0.53} \mathrm{Ga}_{0.47} \mathrm{As} \mathrm{Al}_{0.48} \mathrm{In}_{0.52} \mathrm{As}$ semiconductor superlattice metamaterial, with a $50 \%$ volume fraction of the doped semiconductor with a plasma wavelength of $5 \mu \mathrm{m}$. Blue and red lines correspond to the permittivities parallel and perpendicular to the normal to the layers. Real and imaginary parts of the permittivity are represented, respectively, by solid and dotted lines. For the metamaterial unit cell size of $a \sim 100 \mathrm{~nm}$ in the mid-IR frequency range, this effective medium description is valid as long as $k / k_{0} \ll \lambda_{0} / a \simeq 100$ (see also Fig. 5).
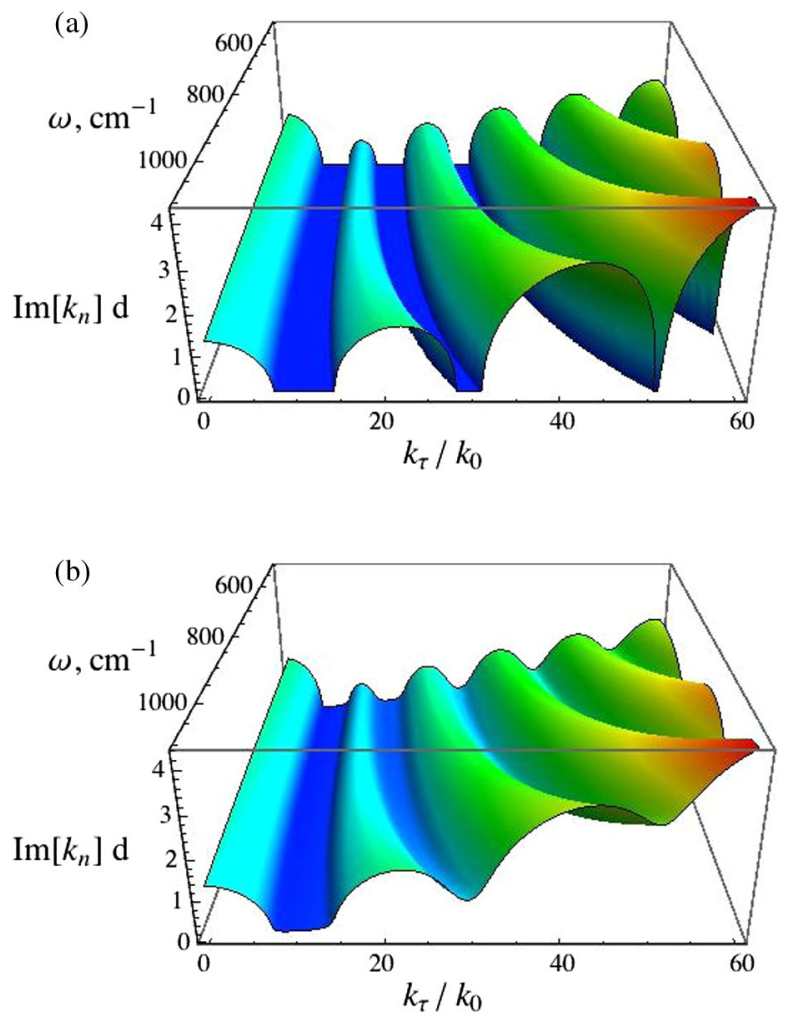

FIG. 4. The dimensionless extinction coefficient $\operatorname{Im}\left[k_{n}\right] d$ vs normalized in-plane momentum $\left(k_{\tau} / k_{0}\right)$ and frequency $\omega$, for a semiconductor $\mathrm{In}_{0.53} \mathrm{Ga}_{0.47} \mathrm{As} \mathrm{Al}_{0.48} \mathrm{In}_{0.52} \mathrm{As} / \mathrm{In}_{0.53} \mathrm{Ga}_{0.47} \mathrm{As}$ hypercrystal, calculated in the lossless limit (a) and for the actual material losses (b). The material parameters for this calculation are described in the caption of Fig. 3 and in the main text. 
(same as in the "isotropic" part of the hypercrystal). The components of the corresponding dielectric permittivity tensor of this semiconductor hyperbolic metamaterial are shown in Fig. 3. While the effective medium theory in Eqs. (7) and (8) clearly leads to a hyperbolic response of this composite, one must ensure that the finite size of the unit cell in the system does not lead to substantial deviations for the wave numbers of interest. To address this issue, in Fig. 5 we compare the isofrequency curves for this composite, calculated with (blue lines) and without (red curves) introducing the effective medium approximation. Note that while for the higher values of the tangential momentum $k_{\tau}>20 k_{0}$ the actual value of $k_{n}$ is no longer nearly indistinguishable from the results of the approximation in Eqs. (7) and (8), the overall behavior of the isofrequency diagram follows the predictions of the effective medium theory.

The presence of the actual material loss results in a nonzero extinction coefficient in the propagation bands, leading to a smaller "contrast" between the propagating and forbidden bands-see Fig. 4(b). However, the extinction coefficient still clearly shows the general "ridge-valley" pattern of the ideal lossless hypercrystal seen in Fig. 4(a). As a result, actual material loss does not fully suppress the hypercrystal behavior.

Note that in the higher-order propagation bands, the typical values of the dimensionless extinction coefficient (9) can substantially exceed unity. As a result, a propagating wave from any of such bands will be totally absorbed at a distance that is barely a small fraction of the free-space wavelength. Therefore, unless one can substantially reduce the effective loss in the hypercrystal, a practical

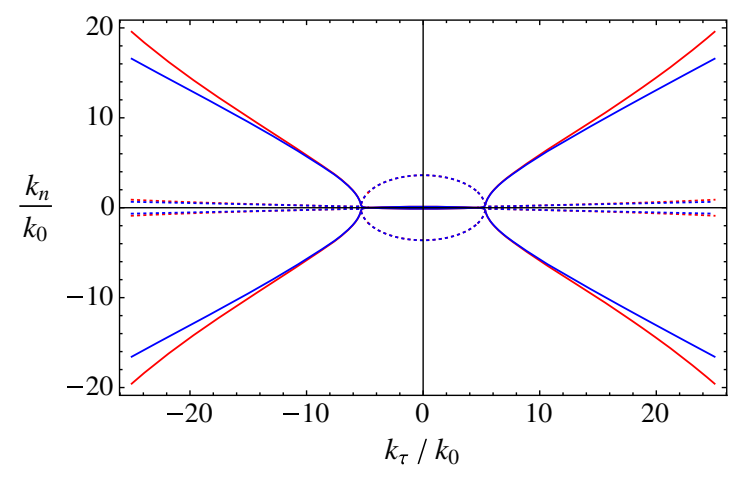

FIG. 5. The isofrequency plot of the semiconductor hyperbolic metamaterial, used in the hypercrystal from Fig. 4, for the free-space wavelength $\lambda=10 \mu \mathrm{m}$ (or equivalently the frequency $\omega=1000 \mathrm{~cm}^{-1}$ ). The metamaterial unit cell is formed by 50-nm-wide layers of insulating $\mathrm{Al}_{0.48} \mathrm{In}_{0.52} \mathrm{As}$ and $n^{+}$-doped $\mathrm{In}_{0.53} \mathrm{Ga}_{0.47}$ As (with the plasma frequency $\lambda_{p}=5 \mu \mathrm{m}$ ). Red and blue colors correspond to the exact solution and the effective medium approximation, respectively. Solid curves represent the real part of the normal to the layer component of the wave vector $k_{n}$, while the dotted lines show $\operatorname{Im}\left[k_{n}\right]$. application of such volume-propagating waves will be very challenging.

Of all the existing realizations of hyperbolic media at optical frequencies, the typical "figure-of-merit" $\operatorname{Re}[\epsilon] / \operatorname{Im}[\epsilon]$ for optical hyperbolic media based on the existing plasmonic and polaritonic materials ranges from unity (e.g., graphene-based hyperbolic metamaterials [48] and graphite [31]) to about 10 (semiconductor [9] and silver-based layered hyperbolic media [21] and sapphire [49]), [50] — with the only exceptions contributed by the nanowire composites [51] where the relatively low volume fraction of the metal (about 10\%) results in a proportionally lower loss $[52,53]$. It is this type of hyperbolic media that holds the most promise for practical applications of the concept of the hypercrystal [54].

However, in addition to the structured spectrum of the "bulk" propagating modes, photonic hypercrystals also support novel surface waves that combine the features of the regular surface plasmons with those of optical Tamm states. Because of the contribution of Bragg scattering to their formation, even with the actual losses in planar hypercrystals, these surface waves show both larger wave numbers and lower loss than their regular surface-plasmon counterparts. It is this behavior of the surface states in photonic hypercrystals that is the main focus of the present paper.

\section{SURFACE WAVES IN PHOTONIC HYPERCRYSTALS}

We consider TM-polarized waves in a planar hypercrystal, formed by layers of an isotropic material with the permittivity $\epsilon_{i}$ and a uniaxial hyperbolic medium with the permittivity tensor

$$
\epsilon=\left(\begin{array}{ccc}
\epsilon_{\tau} & 0 & 0 \\
0 & \epsilon_{\tau} & 0 \\
0 & 0 & \epsilon_{n}
\end{array}\right),
$$

with the corresponding thicknesses $d_{i}$ and $d_{h}$, respectively. Note that at this point we impose no restrictions on the dielectric permittivity $\epsilon_{i}$ of the isotropic component of the hypercrystal - which can be either dielectric $\left(\operatorname{Re}\left[\epsilon_{i}\right]>0\right)$ or metallic/polaritonic $\left(\operatorname{Re}\left[\epsilon_{i}\right]<0\right)$; the corresponding hypercrystals will be referred to, respectively, as "dielectric" and "metallic." In principle, the hypercrystal can also be formed by two distinct anisotropic components, with essentially similar resulting behavior. On the other hand, the extraordinary (TM) polarization here is essential-as TE (or ordinary) waves are only sensitive to the in-plane permittivity $\epsilon_{\tau}$ and generally do not show hyperbolic behavior.

To describe the surface states in the planar hypercrystal, we assume that it occupies the half-space $z>0$ and is terminated at $z=0$ by the interface with the dielectric medium with permittivity $\epsilon_{d}$. This model is exact for natural 
hyperbolic media and assumes that $d_{h} \gg a$ in the case of the hypercrystal unit cell formed from a hyperbolic metamaterial.

\section{A. General theory for planar hypercrystals}

The calculation of the surface waves in such systems is straightforward, extending the standard procedure developed for 1D photonic crystals formed by isotropic components [55] to the more general case of uniaxial anisotropy. For the TM polarization, we obtain

$$
\frac{\epsilon_{1}}{\epsilon_{d}} \frac{\kappa_{d}}{\kappa_{1}}=\frac{\frac{1}{\lambda}-T_{11}-T_{12}}{\frac{1}{\lambda}-T_{11}+T_{12}},
$$

where $k_{0} \equiv \omega / c$ is the free-space wave number, $\kappa_{d}$ is the field decay rate in the dielectric at $z<0$,

$$
\kappa_{d}=\sqrt{k_{\tau}^{2}-\epsilon_{d} k_{0}^{2}}
$$

and the permittivity $\epsilon_{1}$ and the decay constant $\kappa_{1}$ correspond to the parameters of the "terminating" layer of the hypercrystal that interfaces the surrounding medium: If the terminating layer is isotropic, $\epsilon_{1}=\epsilon_{i}$ and $\kappa_{1}=\kappa_{i}$, while for the hyperbolic terminating layer, $\epsilon_{1}=\epsilon_{\tau}$ and $\kappa_{1}=-i k_{h}$. Here, the wave number in the hyperbolic medium $k_{h}$ and the field decay rate in the isotropic part of the hypercrystal unit cell $\kappa_{i}$ are given by

$$
k_{h}=\sqrt{\epsilon_{\tau} k_{0}^{2}-\left(\epsilon_{\tau} / \epsilon_{n}\right) k_{\tau}^{2}}, \quad \kappa_{i}=\sqrt{k_{\tau}^{2}-\epsilon_{i} k_{0}^{2}} .
$$

$T_{11}$ and $T_{12}$ are the components of the $2 \times 2$ transfer matrix

$$
T \equiv\left(\begin{array}{ll}
T_{11} & T_{12} \\
T_{21} & T_{22}
\end{array}\right)
$$

for the unit cell of the hypercrystal [55], and $\lambda$ is its eigenvalue with the absolute value larger than 1 .

Since the determinant of a unit cell transfer matrix in a periodic system (even in the presence of material absorption) is equal to 1 ,

$$
\operatorname{det}[T] \equiv T_{11} T_{22}-T_{12} T_{21}=1,
$$

Eq. (11) can also be equivalently expressed as

$$
-\frac{\epsilon_{1}}{\epsilon_{d}} \frac{\kappa_{d}}{\kappa_{1}}=\frac{\frac{1}{\lambda}-T_{22}-T_{21}}{\frac{1}{\lambda}-T_{22}+T_{21}} .
$$

Note that the system only supports surface states if the trace of the $T$ matrix

$$
|\operatorname{Tr}[T]| \equiv\left|T_{11}+T_{22}\right|>2,
$$

which for $\operatorname{det}[T]=1$ is a necessary and sufficient condition for the existence of an eigenvalue with the modulus $|\lambda|>1$. The physical meaning of this requirement can be uncovered in the lossless limit, where Eq. (16) implies that the surface states in the hypercrystal, just as the regular optical Tamm states, are confined to the band gaps of the volume-propagating modes.

From Eq. (11), we obtain

$$
\frac{1}{\lambda}=T_{11}+T_{12} \frac{\epsilon_{d} \kappa_{1}+\epsilon_{1} \kappa_{d}}{\epsilon_{d} \kappa_{1}-\epsilon_{1} \kappa_{d}} .
$$

For an infinite hypercrystal, the transfer matrix $T$ that, by definition, relates the amplitudes of the "left"-and "right"-propagating waves in different unit cells, can be calculated either in the hyperbolic or in the isotropic material components. Equations (11) and (15), however, remove this uncertainly, as the $T$-matrix components there must be calculated in the medium that forms the "terminal layer" of the hypercrystal, which is in direct contact with the surrounding medium.

When the hypercrystal is terminated at the hyperbolic layer (see Fig. 6), using a a straightforward generalization of the approach of Ref. [55] to the case of anisotropic material components, for the unit cell transfer matrix, we obtain

$$
\begin{aligned}
T_{11}= & {\left[\cosh \left(\kappa_{i} d_{i}\right)+\frac{i}{2}\left(\frac{k_{h}}{\kappa_{i}} \frac{\epsilon_{i}}{\epsilon_{\tau}}-\frac{\kappa_{i}}{k_{h}} \frac{\epsilon_{\tau}}{\epsilon_{i}}\right) \sinh \left(\kappa_{i} d_{i}\right)\right] } \\
& \times \exp \left(i k_{h} d_{h}\right), \\
T_{12}= & -\frac{i}{2}\left(\frac{k_{h}}{\kappa_{i}} \frac{\epsilon_{i}}{\epsilon_{\tau}}+\frac{\kappa_{i}}{k_{h}} \frac{\epsilon_{\tau}}{\epsilon_{i}}\right) \sinh \left(\kappa_{i} d_{i}\right) \exp \left(-i k_{h} d_{h}\right),
\end{aligned}
$$
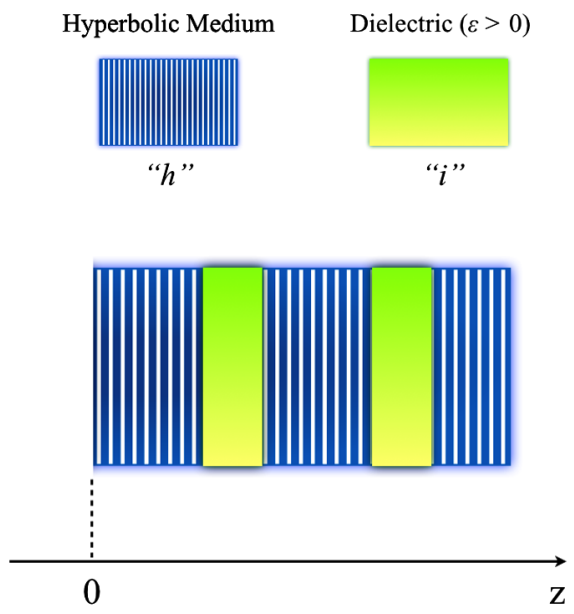

FIG. 6. The interface of a "dielectric" hypercrystal with air, with the former "terminated" at the hyperbolic layer. The letters in quotation marks represent the subscripts used in Sec. III for the corresponding materials. 


$$
\begin{aligned}
T_{21}= & \frac{i}{2}\left(\frac{k_{h}}{\kappa_{i}} \frac{\epsilon_{i}}{\epsilon_{\tau}}+\frac{\kappa_{i}}{k_{h}} \frac{\epsilon_{\tau}}{\epsilon_{i}}\right) \sinh \left(\kappa_{i} d_{i}\right) \exp \left(i k_{h} d_{h}\right), \\
T_{22}= & {\left[\cosh \left(\kappa_{i} d_{i}\right)-\frac{i}{2}\left(\frac{k_{h}}{\kappa_{i}} \frac{\epsilon_{i}}{\epsilon_{\tau}}-\frac{\kappa_{i}}{k_{h}} \frac{\epsilon_{\tau}}{\epsilon_{i}}\right) \sinh \left(\kappa_{i} d_{i}\right)\right] } \\
& \times \exp \left(-i k_{h} d_{h}\right) .
\end{aligned}
$$

On the other hand, when it is the isotropic layer that forms the surface of the hypercrystal (see, e.g., Fig. 7),

$$
\begin{aligned}
T_{11}= & {\left[\cos \left(k_{h} d_{h}\right)+\frac{1}{2}\left(\frac{k_{h}}{\kappa_{i}} \frac{\epsilon_{i}}{\epsilon_{\tau}}-\frac{\kappa_{i}}{k_{h}} \frac{\epsilon_{\tau}}{\epsilon_{i}}\right) \sin \left(k_{h} d_{h}\right)\right] } \\
& \times \exp \left(-\kappa_{i} d_{i}\right), \\
T_{12}= & \frac{1}{2}\left(\frac{k_{h}}{\kappa_{i}} \frac{\epsilon_{i}}{\epsilon_{\tau}}+\frac{\kappa_{i}}{k_{h}} \frac{\epsilon_{\tau}}{\epsilon_{i}}\right) \sin \left(k_{h} d_{h}\right) \exp \left(\kappa_{i} d_{i}\right), \\
T_{21}= & -\frac{1}{2}\left(\frac{k_{h}}{\kappa_{i}} \frac{\epsilon_{i}}{\epsilon_{\tau}}+\frac{\kappa_{i}}{k_{h}} \frac{\epsilon_{\tau}}{\epsilon_{i}}\right) \sin \left(k_{h} d_{h}\right) \exp \left(-\kappa_{i} d_{i}\right), \\
T_{22}= & {\left[\cos \left(k_{h} d_{h}\right)-\frac{1}{2}\left(\frac{k_{h}}{\kappa_{i}} \frac{\epsilon_{i}}{\epsilon_{\tau}}-\frac{\kappa_{i}}{k_{h}} \frac{\epsilon_{\tau}}{\epsilon_{i}}\right) \sin \left(k_{h} d_{h}\right)\right] } \\
& \times \exp \left(\kappa_{i} d_{i}\right) .
\end{aligned}
$$

Note that in both cases, the trace of the transfer matrix (which is uniquely defined by the corresponding eigenvalues)

$$
\begin{aligned}
\operatorname{Tr}[T]= & 2 \cos \left(k_{h} d_{h}\right) \cosh \left(\kappa_{i} d_{i}\right) \\
& +\left(\frac{\kappa_{i}}{k_{h}} \frac{\epsilon_{\tau}}{\epsilon_{i}}-\frac{k_{h}}{\kappa_{i}} \frac{\epsilon_{i}}{\epsilon_{\tau}}\right) \sin \left(k_{h} d_{h}\right) \sinh \left(\kappa_{i} d_{i}\right)
\end{aligned}
$$

yields the normal to the layer component of the wave number $k_{n}$ :

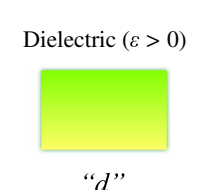

"d"

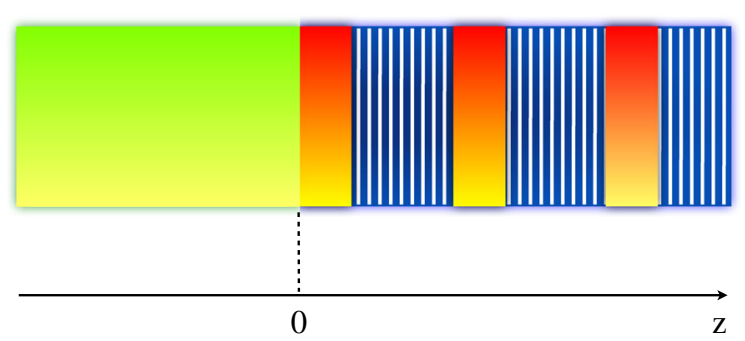

FIG. 7. The interface of a "metallic" hypercrystal and a dielectric. The hypercrystal is terminated at the metallic layer. The symbols in quotation marks show the corresponding subscripts in Sec. III.

$$
\operatorname{Tr}[T]=\cos \left[k_{n}\left(d_{i}+d_{h}\right)\right] .
$$

Substituting Eqs. (17)-(25) into Eq. (15), we find

$$
\frac{k_{h} \epsilon_{\tau}\left(\epsilon_{d}^{2} \kappa_{i}^{2}-\epsilon_{i}^{2} \kappa_{d}^{2}\right)}{\tan \left(k_{h} d_{h}\right)}-\frac{\kappa_{i} \epsilon_{i}\left(\epsilon_{\tau}^{2} \kappa_{d}^{2}+\epsilon_{d}^{2} k_{h}^{2}\right)}{\tanh \left(\kappa_{i} d_{i}\right)}= \pm \kappa_{d} \epsilon_{d}\left(\epsilon_{\tau}^{2} \kappa_{i}^{2}+\epsilon_{i}^{2} k_{h}^{2}\right) .
$$

Here, the positive sign in the right-hand side of Eq. (28) corresponds to a hypercrystal where the "last" layer is isotropic, while the negative sign describes the case of the interface formed by the hyperbolic phase of the composite.

Equation (28) can be considered as the fundamental equation for the surface states in a hypercrystal. However, while it no longer explicitly depends on the eigenvalue $\lambda$, this equation is still limited to the case of $|\lambda|>1$ and should therefore be considered together with the inequality (16). Note that, while the actual dispersion of the surface state does depend on which of the layers forming the hypercrystal interfaces with the surrounding medium, the condition $|\lambda|>1$ does not. In other words, if a hypercrystal supports the surface wave for a given frequency when the terminating layer is isotropic, it will also support the corresponding surface wave when the terminating layer is hyperbolic, and vice versa.

In the lossless limit, the requirement of $|\lambda|>1$ confines the surface waves to the band gaps of the hypercrystal, just as in the case of the conventional Tamm states in regular photonic crystals. However, in contrast to the latter, the hypercrystals support band gaps at $k_{\tau} \gg k_{0}$, which leads to the immediate conclusion that, aside from the zero-order mode, the surface states in hypercrystals generally possess high wave numbers. By continuity, this conclusion should remain valid even in the presence of material loss. Mathematically, this is easily seen from Eq. (28), which for high-order modes, implies that $\kappa d=\mathcal{O}(1)$, leading to $k_{\tau} \sim 1 / d \gg k_{0}$.

In the limit $k_{\tau} \gg k_{0} \equiv \omega / c$, we find

$$
\frac{A_{h}}{\tan \left(k_{\tau} d_{*}\right)}= \pm 1+\frac{A_{i}}{\tanh \left(k_{\tau} d_{i}\right)}
$$

where

$$
d_{*} \equiv d_{h} \sqrt{-\epsilon_{\tau} / \epsilon_{n}}
$$

and the coefficients $A_{h}$ and $A_{i}$ are defined by the permittivites $\epsilon_{\tau}, \epsilon_{n}, \epsilon_{i}$, and $\epsilon_{d}$ :

$$
\begin{aligned}
A_{h} & =\frac{\sqrt{-\epsilon_{\tau} \epsilon_{n}}}{\epsilon_{d}} \frac{\epsilon_{i}^{2}-\epsilon_{d}^{2}}{\epsilon_{i}^{2}-\epsilon_{\tau} \epsilon_{n}}, \\
A_{i} & =\frac{\epsilon_{i}}{\epsilon_{d}} \frac{\epsilon_{d}^{2}-\epsilon_{\tau} \epsilon_{n}}{\epsilon_{i}^{2}-\epsilon_{\tau} \epsilon_{n}}
\end{aligned}
$$


Equation (29) should be solved within the bounds set by the inequality (16), which in this limit $\left(k_{\tau} \gg k_{0}\right)$, reduces to

$$
\begin{gathered}
\mid \cos \left(k_{\tau} d_{*}\right) \cosh \left(k_{\tau} d_{i}\right)+\sin \left(k_{\tau} d_{*}\right) \sinh \left(k_{\tau} d_{i}\right) \\
\quad \times \frac{1}{2}\left(\sqrt{-\frac{\epsilon_{n}}{\epsilon_{\tau}}} \frac{\epsilon_{\tau}}{\epsilon_{i}}-\sqrt{-\frac{\epsilon_{\tau}}{\epsilon_{n}}} \frac{\epsilon_{i}}{\epsilon_{\tau}}\right) \mid>1 .
\end{gathered}
$$

Note that, depending on the nature of the surrounding material and material composition, as well as the structural characteristics of the hypercrystal, the resulting surface states can be both right- and left-handed (corresponding to forward- and backward-propagating modes); in the following sections, we will present the examples of both types of behavior. This is similar to the situation found in "traditional" photonic crystals where surface modes can be either forward or backward [56].

While the general theory of the present subsection can be used for both "dielectric" (with the isotropic phase having the dielectric response: $\operatorname{Re}\left[\epsilon_{i}\right]>0$, as in Fig. 6) and "metallic" (when the permittivity of the isotropic component is negative: $\operatorname{Re}\left[\epsilon_{i}\right]<0$; see, e.g., Fig. 7) hypercrystals, the qualitative difference in the electromagnetic response of the isotropic phase has a profound effect on the surface states in these two classes of composites. This will be discussed in detail in the next two sections.

\section{B. Dielectric hypercrystals}

As an example of a dielectric hypercrystal, we consider a composite formed by layers of silicon as the isotropic dielectric, and sapphire as the natural hyperbolic medium. Note that, in contrast to the composites that use metamaterial realizations of the hyperbolic media, this hypercrystal is not subject to the lower limit of the inequality (3) [see also Eq. (4) and the discussion that immediately precedes it) and therefore offers an essentially unlimited range of supported wave numbers, up to the inverse atomic size.

Because of a strong anisotropy of its crystal structure and high-quality optical phonon resonances, monocrystalline sapphire shows hyperbolic behavior in several frequency bands in the mid-IR range. This is illustrated in Fig. 8(a) which summarizes the experimental data for the dielectric permittivity of sapphire from Ref. [23].

In particular, the range around the frequency of $500 \mathrm{~cm}^{-1}$ (corresponding to the free-space wavelength of $20 \mu \mathrm{m}$ ), shown in Fig. 8(b), supports a low-loss hyperbolic band with negative permittivity along the symmetry axis of the crystal [red line in Fig. 8(b)] and positive permittivity in the plane perpendicular to the symmetry axis [blue curve in Fig. 8(b)] for $485 \mathrm{~cm}^{-1}<\omega<510 \mathrm{~cm}^{-1}$.

Silicon, on the other hand, is transparent in this frequency range, with the dielectric permittivity $\epsilon_{\mathrm{Si}} \approx 11.6$ [57]. Since this exceeds the magnitudes of both components of the sapphire permittivity tensor, within the (a)

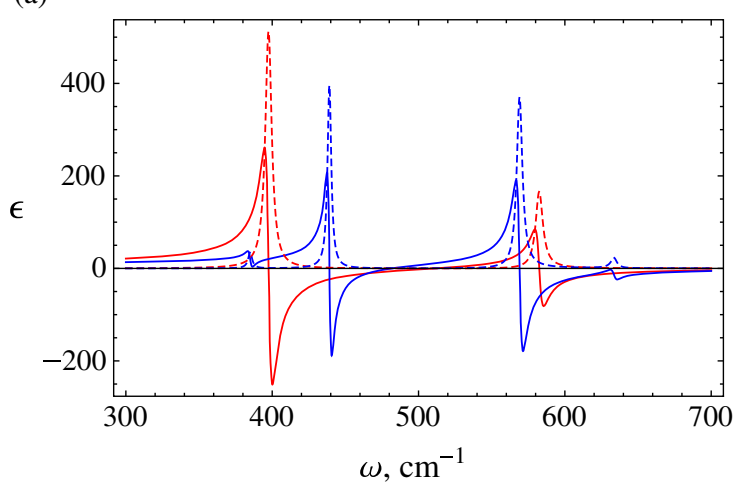

(b)

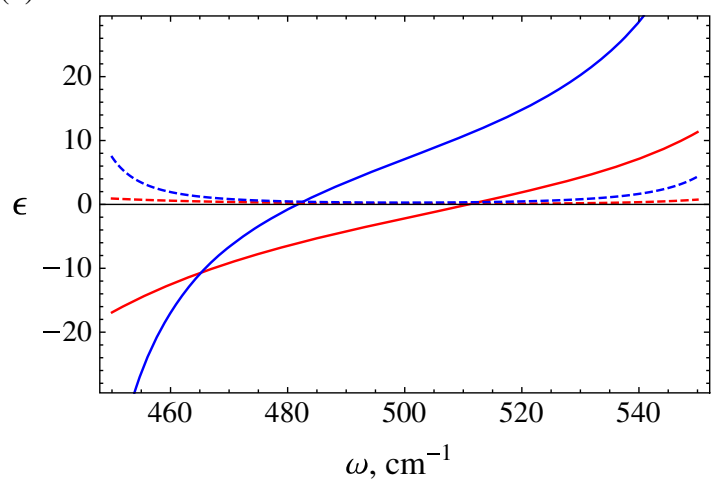

FIG. 8. Panel (a): the dielectric permittivity tensor of sapphire monocrystal in the Restrahlen band, from the measurements of Ref. [23]. Panel (b) shows the range near the free-space wavelength of $20 \mu \mathrm{m}$ that supports a low-loss hyperbolic band. Red and blue lines correspond, respectively, to the permittivities parallel and perpendicular to the $C$ axis of the sapphire crystal. Real and imaginary parts of the permittivity are represented by solid and dotted lines.

effective medium approximation, the silicon-sapphire hypercrystal with equal volume fractions of both components is characterized by a dielectric permittivity tensor where all diagonal components have positive real parts.

With the thicknesses of the silicon and sapphire layers $d_{i}=d_{h}=1 \mu \mathrm{m}$ forming the unit cell that is an order of magnitude smaller than the free-space wavelength of $20 \mu \mathrm{m}$ (corresponding to $\omega=500 \mathrm{~cm}^{-1}$ ), one would naively expect the hypercrystal to behave as an effective anisotropic dielectric that does not support any high- $k$ surface-plasmonlike states. The actual presence of such surface waves in this system is yet another example of the failure of the effective medium approximation in photonic hypercrystals.

For the interface of air and a silicon-sapphire hypercrystal with $d_{h}=d_{i}=1 \mu \mathrm{m}$, with the sapphire symmetry axis normal to its layers, Eqs. (28) and (16) yield a sequence of surface states. In Fig. 9, we show the first three of these, at the frequency $\omega=500 \mathrm{~cm}^{-1}$. An interesting feature of these waves is that they are "left-handed," with the average energy flow along the surface opposite to 


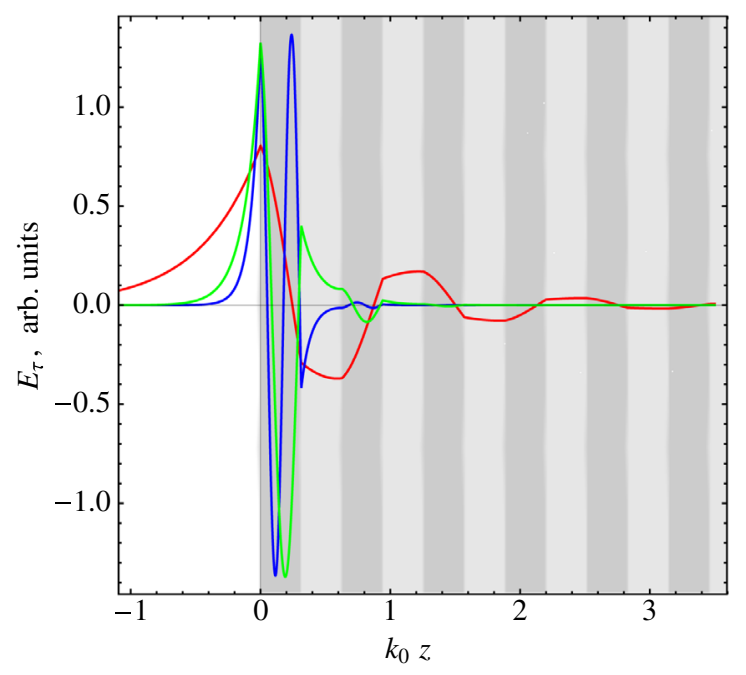

FIG. 9. The profiles of the tangential electric field for the first three surface states at the interface of air and a silicon-sapphire hypercrystal. The unit cell of the hypercrystal is formed by $1-\mu \mathrm{m}-$ wide layers of silicon and sapphire, with the $C$ axis of sapphire normal to the plane of the layers. The frequency is $500 \mathrm{~cm}^{-1}$. The dark-gray regions correspond to sapphire, and light-gray areas represent silicon.

the direction of the in-plane momentum $\mathbf{k}_{\tau}$. This, however, is not surprising since-just as the free-electron wavefunctions in a solid can be treated as superpositions of the localized atomic eigenstates [40] - the electromagnetic waves in a hypercrystal are formed by the propagating modes of coupled hyperbolic waveguides, which for $\operatorname{Re}\left[\epsilon_{\tau}\right]>0, \operatorname{Re}\left[\epsilon_{n}\right]<0$ (corresponding to this hyperbolic band in sapphire), are known to be left-handed [41].

Figures 10 and 11 show the corresponding dispersion diagrams for the surface states in the silicon-sapphire hypercrystal, for the whole hyperbolic band in sapphire that is near the free-space wavelength of $20 \mu \mathrm{m}$.

In Fig. 10, we plot $\omega\left(k_{\tau}\right)$ for the silicon-sapphire hypercrystal surface waves when the material loss is neglected. Note that all these modes are backwardspropagating (or left-handed) since the group velocity $v_{g} \equiv \partial \omega / \partial k_{\tau}$ is negative.

The lossless limit of Fig. 10 also shows the divergence $k_{\tau} \rightarrow \infty$ as the (positive) in-plane permittivity in the hyperbolic layers $\epsilon_{\tau}$ approaches zero. Indeed, as follows from Eqs. (29)-(32), for a dielectric hypercrystal in the limit $\epsilon_{\tau} \rightarrow 0$, we find the "resonance"

$$
k_{\tau}=\frac{\pi}{d_{h}} \sqrt{-\frac{\epsilon_{n}}{\epsilon_{\tau}}} m \rightarrow \infty
$$

where $m$ is an integer. Physically, this limit corresponds to most of the energy of the surface state localized in the first hyperbolic layer near the interface. This divergence is, however, removed when the actual material losses are taken into account-see Fig. 11.

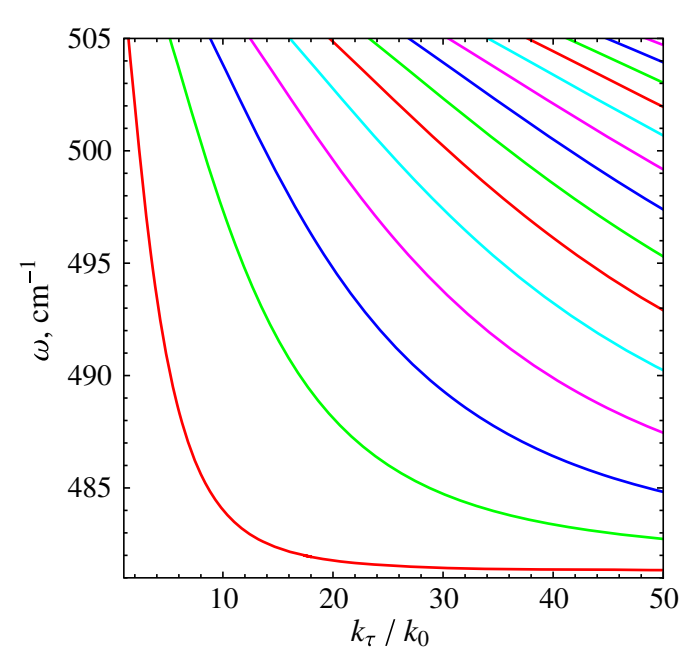

FIG. 10. The wave dispersion $\omega\left(k_{\tau}\right)$ for the surface states at the interface of a silicon-sapphire hypercrystal with air in the zero loss limit; the unit cell of the composite is formed by silicon and sapphire layers of equal widths, $d_{h}=d_{i}=1 \mu \mathrm{m}$. Note that for all modes, (i) $k_{\tau}>k_{0}$ (as the coordinate axis origin corresponds to $k_{\tau} / k_{0}=1$ ), so that they are outside the free-space "light cone" $k_{\tau}<k_{0}$ and therefore cannot directly couple to the air, and (ii) the group velocity $v_{g}<0$.

The comparison of Figs. 10 and 11 also shows that, away from the $\epsilon_{\tau} \rightarrow 0$ resonance point at $\omega \approx 481 \mathrm{~cm}^{-1}$ (see Fig. 8), the material loss in sapphire does not have a strong effect on the surface states, which show both the large wave numbers and relatively long propagation distances (inversely proportional to the imaginary part of the wave number shown in Fig. 11 by dotted lines).

This behavior is further illustrated in Fig. 12, where we plot the standard "figure of merit" for surface states,

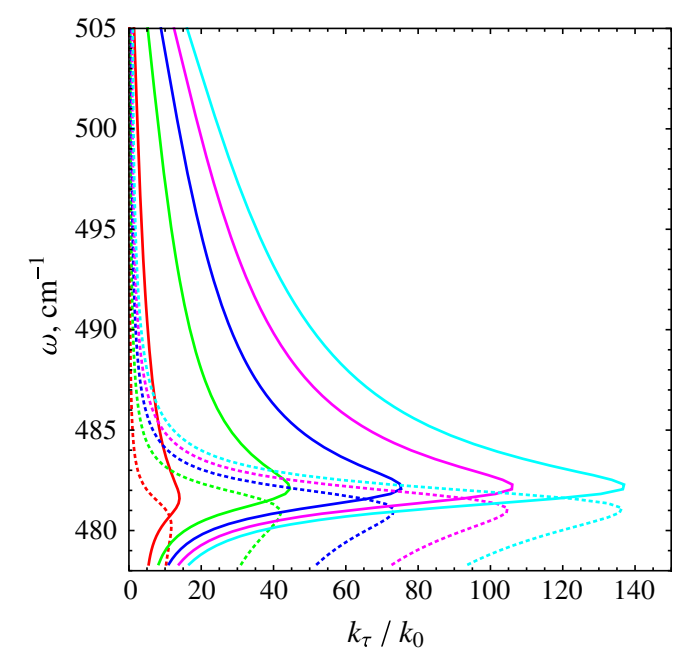

FIG. 11. The dispersion diagrams for the first five surface waves at the interface of air and the silicon-sapphire hypercrystal (same as in Figs. 9 and (10)), with the actual loss. The solid and dashed lines correspond, respectively, to the real and imaginary parts of the in-plane momentum $k_{\tau}$. 


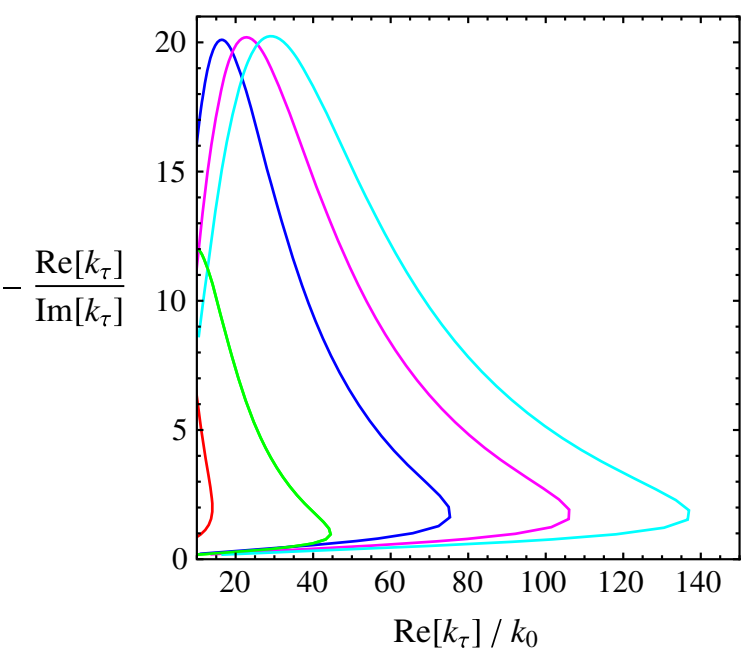

FIG. 12. The figure of merit, $\mathrm{FOM}=-\operatorname{Re}\left[k_{\tau}\right] / \operatorname{Im}\left[k_{\tau}\right]$, as a function of the real part of the in-plane momentum $k_{\tau}$ for the silicon-sapphire hypercrystal and air interface. Note that as the group velocity for these modes $v_{g} \equiv \partial \omega / \partial k_{\tau}<0$ (see Fig. 10), the surface waves in this system are left-handed (with the energy flow along the surface opposite to the in-plane momentum) so that $\operatorname{Im}\left[k_{\tau}\right]<0$, thus the negative sign in the figure of merit.

$\mathrm{FOM}=\left|\operatorname{Re}\left[k_{\tau}\right] / \operatorname{Im}\left[k_{\tau}\right]\right|$, which describes the propagation distance of the wave vs the real part of the dimensionless inplane wave number $k_{\tau} / k_{0}$. In contrast to the situation with the regular surface plasmons, where large wave numbers are achieved at the expense of reduced propagation distance and vice versa, Fig. 12 demonstrates that surface waves in a hypercrystal allow for both long propagation distances (FOM $\sim 10)$ and high wave numbers $\left(k_{\tau} \sim 50 k_{0}\right)$ at the same time.

\section{Metallic hypercrystals}

As an example of a "metallic" hypercrystal, we consider the composite formed by semiconductor hyperbolic metamaterial and a doped semiconductor - the material system that we have already used in describing the general concept of the hypercrystal (see, e.g., Fig. 4). While also designed for the mid-IR range, just as the silicon-sapphire hypercrystal described earlier, the semiconductor aluminumindium-gallium-arsenide "platform" offers the capability of growing multilayer composites with atomic-level precision, leading to high-quality hypercrystals. This is particularly important for high-wave-number states, which by their very nature, are more sensitive to short-range disorder.

The unit cell of the hypercrystal described in the present section consists of a $1.9-\mu \mathrm{m}$-wide layer of hyperbolic semiconductor metamaterial, followed by $100 \mathrm{~nm}$ of an $n^{+}$-doped $\mathrm{In}_{0.53} \mathrm{Ga}_{0.47}$ As semiconductor with a plasma frequency of $5 \mu \mathrm{m}$. The semiconductor hyperbolic metamaterial used in this example corresponds to the wellstudied system [9] of interleaving layers of the dielectric $\mathrm{Al}_{0.48} \operatorname{In}_{0.52} \mathrm{As}$ and $n^{+}$-doped $\operatorname{In}_{0.53} \mathrm{Ga}_{0.47}$ As semiconductor.
Note that both the hyperbolic and the isotropic parts of the hypercrystal use the same "metallic" component, which would greatly simplify the fabrication of such composites.

As expected, this system supports a sequence of distinct surface states (note the difference from the surface-plasmon polaritons in metals and surface waves in hyperbolic media, where the system supports a single surface state). In Fig. 13, we plot the first three solutions for the surface states at the interface of the semiconductor hypercrystal and dielectric $\mathrm{Al}_{0.48} \mathrm{In}_{0.52} \mathrm{As}$. Note that in this example, $v_{g} \equiv \partial \omega / \partial k_{\tau}>0$, so these modes are forward-propagating (or right-handed).

Aside from the zeroth-order mode whose dispersion is nearly identical to the regular surface plasmon on the interface between the doped semiconductor and the external dielectric, the surface waves supported by the system have frequencies approaching the lower-band bounds in the spectrum of the bulk-propagating modes of the hypercrystal.

The presence of the actual material loss limits the maximum values of the surface-state wave numbers while at the same time extending their existence to frequencies beyond the maximum values from the lossless case- see Fig. 14. This is exactly what is expected for surface states in plasmonic systems. However, compared to the regular surface plasmons, here we find a dramatic increase of the maximum allowed momentum.

The limits on the wave numbers and the propagation distance of the surface waves in hypercrystals are further illustrated in Fig. 15, where we plot the standard figure of merit [58] $\operatorname{Re}[k] / \operatorname{Im}[k]$ proportional to the propagation distance in units of the actual wavelength vs the

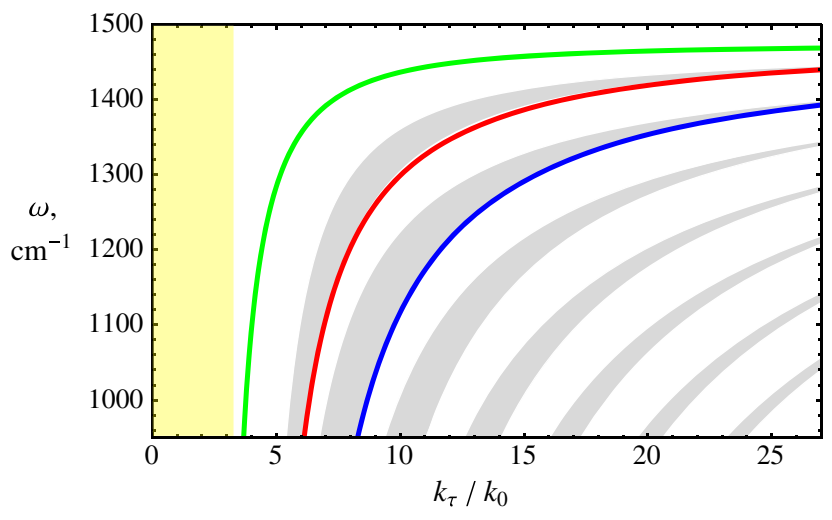

FIG. 13. The surface states at the interface of InGaAs:AlInAs semiconductor-based hypercrystal and dielectric $\left(\mathrm{Al}_{0.48} \mathrm{In}_{0.52} \mathrm{As}\right)$, in the lossless limit. Gray areas correspond to the volumepropagating bands of the hypercrystal. The color code represents surface states of zero (green), one (red), and two (blue) orders. The unit cell of the hypercrystal is formed by $1.9-\mu \mathrm{m}$-thick semiconductor hyperbolic metamaterial, and a 100-nm-wide layer of $n^{+}$-doped $\operatorname{In}_{0.53} \mathrm{Ga}_{0.47} \mathrm{As}$ (with a plasma wavelength of $5 \mu \mathrm{m}$ ). The yellow band marks the light cone of the dielectric covering the hypercrystal. 


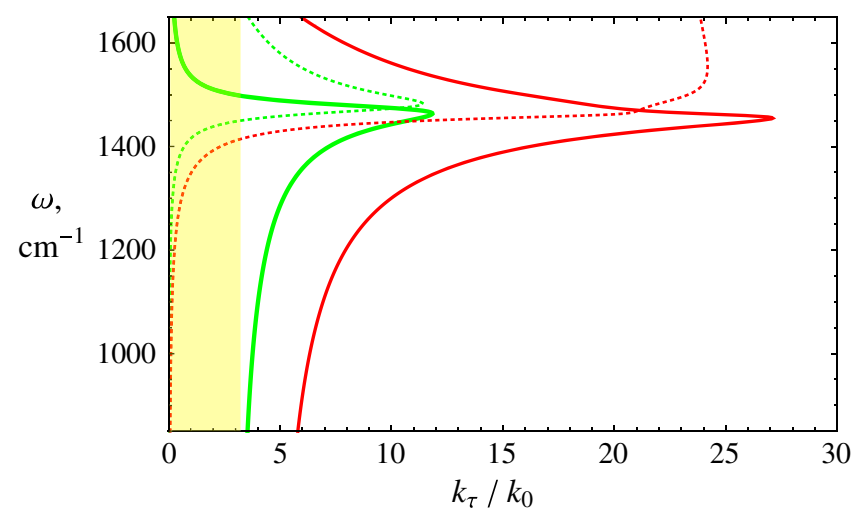

FIG. 14. Surface states at the interface of a semiconductor hypercrystal with air (same as in Fig. 13), taking into account the actual material absorption, for orders zero (green) and one (red). Solid and dashed lines correspond, respectively, to the real and imaginary parts of the in-plane momentum of the surface modes, while the translucent yellow region corresponds to the light cone of the dielectric that borders the hypercrystal $k_{\tau}<\sqrt{\epsilon_{d}} \omega / c$.

confinement/wavelength compression factor $k_{\tau} / k_{0}$. There, the first- and second-order modes (red and blue curves, respectively) are compared to the regular surface plasmon (black line) at the interface of the same materials as those forming the hypercrystal (doped InGaAs as the "metal" and AlInAs as the dielectric). Note that, with the same material loss, these "hyperplasmons" show more than an order-ofmagnitude improvement in propagation distance at the

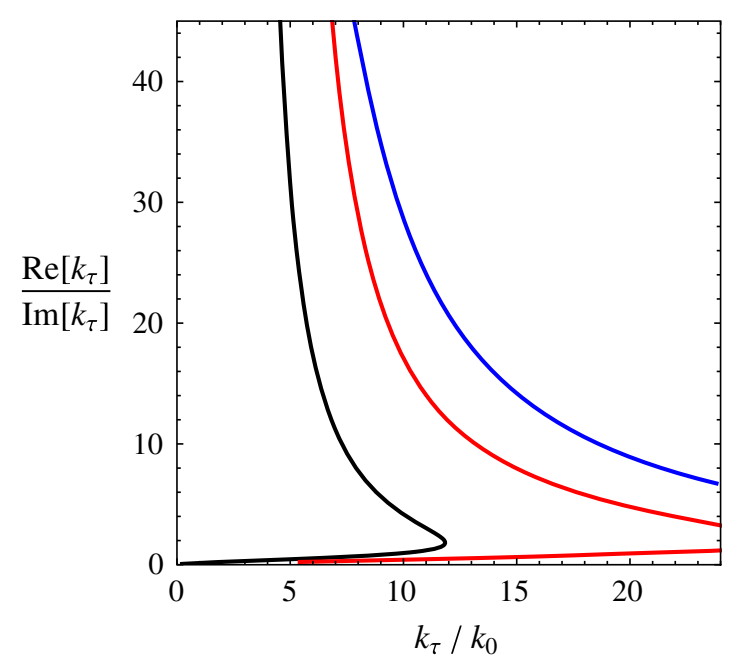

FIG. 15. Propagation distance in units of the local wavelength $\left(\operatorname{Re}\left[k_{\tau}\right] / \operatorname{Im}\left[k_{\tau}\right]\right)$ vs the dimensionless wave number (normalized to the one in free space) for the surface states of the first (red curve) and second (blue line) order in the semiconductor hypercrystal (same as in Figs. 13 and 14), compared to that of the standard surface plasmon at the metal-dielectric interface (black curve). The surface plasmon corresponds to the interface of $n^{+}$doped $\mathrm{In}_{0.53} \mathrm{Ga}_{0.47}$ As and dielectric $\mathrm{Al}_{0.48} \mathrm{In}_{0.52} \mathrm{As}$ [9] - the same materials as those that form the hypercrystal. same value of $k_{\tau}$, and more than a factor of 3 enhancement of the maximum accessible wave number.

\section{CONCLUSIONS}

The properties of electromagnetic surface waves in hypercrystals make these artificial media an ideal "playground" for what so far has been called the surface-plasmon physics. With enhancement in both the propagation distance and in the wave numbers, the use of the hyperplasmons introduced in the present work would lead to substantial enhancements in all possible applications in this field-from near-field imaging and superresolution to surface-enhanced Raman spectroscopy and surface-plasmon resonance sensing and detection.

Furthermore, the photonic hypercrystal, the unifying concept, until recently, of mutually exclusive limits of metamaterials and photonic crystals, dramatically extends the accessible "phase space" in electromagnetic material response and therefore has the potential to find many other applications in nanophotonics. While the hypercrystal represents an extra level in the device complexity, its fabrication does not require anything beyond the standard methods used in the metamaterial community $[1,9]$.

\section{ACKNOWLEDGMENTS}

This work was partially supported by the NSF Center for Photonic and Multiscale Nanomaterials, ARO MURI Grant No. 56154-PH-MUR (W911NF-09-1-0539), and the Gordon and Betty Moore Foundation. The author would like to thank I. Aladinski for helpful discussions.

[1] W. Cai and V. M. Shalaev, Optical Metamaterials (Springer, New York, NY, 2010).

[2] J. D. Joannopoulos, S. G. Johnson, J. N. Winn, and R. D. Meade, Photonic Crystals: Molding the Flow of Light (Princeton University Press, Princeton, NJ, 2008).

[3] J. Valentine, S. Zhang, T. Zentgraf, E. Ulin-Avila, D. A. Genov, G. Bartal, and X. Zhang, Three-Dimensional Optical Metamaterial with a Negative Refractive Index, Nature (London) 455, 376 (2008).

[4] V. M. Shalaev, Optical Negative Refractive-Index Metamaterials, Nat. Photonics 1, 41 (2007).

[5] E. Yablonovitch, Inhibited Spontaneous Emission in Solid-State Physics and Electronics, Phys. Rev. Lett. 58, 2059 (1987).

[6] S. John, Strong Localization of Photons in Certain Disordered Dielectric Superlattices, Phys. Rev. Lett. 58, 2486 (1987).

[7] Z. Jacob, L. V. Alekseyev, and E. E. Narimanov, Optical Hyperlens: Far-Field Imaging Beyond the Diffraction Limit, Opt. Express 14, 8247 (2006).

[8] D. R. Smith and D. Schurig, Electromagnetic Wave Propagation in Media with Indefinite Permittivity and Permeability Tensors, Phys. Rev. Lett. 90, 077405 (2003). 
[9] A. J. Hoffman, L. V. Alekseyev, S. S. Howard, K. J. Franz, D. Wasserman, V. A. Podolskiy, E. E. Narimanov, D. L. Sivco, and C. Gmachl, Negative Refraction in Semiconductor Metamaterials, Nat. Mater. 6, 946 (2007).

[10] Y. Xiang, X. Dai, and S. Wen, Omnidirectional Gaps of One-Dimensional Photonic Crystals Containing Indefinite Metamaterials, J. Opt. Soc. Am. B 24, 2033 (2007).

[11] T. Pan, G. Xu, T. Zang, and L. Gao, Goos-Hänchen Shift in One-Dimensional Photonic Crystals Containing Uniaxial Indefinite Medium, Phys. Status Solidi B 246, 1088 (2009).

[12] Surface Plasmon Resurrection (editorial), Nat. Photonics 6 , 707 (2012).

[13] I. Tamm, On the Possible Bound States of Electrons on a Crystal Surface, Phys. Z. Soviet Union 1, 733 (1932).

[14] P. Yeh, A. Yariv, and A. Y. Cho, Optical Surface Waves in Periodic Layered Media, Appl. Phys. Lett. 32, 104 (1978).

[15] A. V. Kavokin, I. A. Shelykh, and G. Malpuech, Lossless Interface Modes at the Boundary between Two Periodic Dielectric Structures, Phys. Rev. B 72, 233102 (2005).

[16] Z. Chen, P. Han, C. W. Leung, Y. Wang, M. Hu, and Y. Chen, Study of Optical Tamm States Based on the Phase Properties of One-Dimensional Photonic Crystals, Opt. Express 20, 21618 (2012).

[17] W. Shochkley, On the Surface States Associated with a Periodic Potential, Phys. Rev. 56, 317 (1939).

[18] N. Malkova and C.Z. Ning, Shockley and Tamm Surface States in Photonic Crystals, Phys. Rev. B 73, 113113 (2006).

[19] The distinction between Tamm and Shockley states based on the strength of the coupling between the original "building blocks" of the medium (such as atoms in a solid or planar dielectric waveguides forming a one-dimentional photonic crystal), which is sometimes found in the literature (see, e.g., Ref. [18]), is somewhat artificial and certainly goes against the original intent of Shockley who generalized Tamm's approach beyond the simple Kronig-Penny model with a single interface. In Shockley's own words, the objective of his paper [17] was to find how "Tamm's levels" "originate from the atomic levels as the crystal is conceived of as being formed by varying the lattice constant from infinity to a finite value." With Tamm's original publication [13] being a simple two-page summary with only a single equation and no figures, rather than a full-length paper, it was primarily Shockley's work [17] that brought the actual understanding of surface states in periodic potentials to the research community.

[20] G. W. Milton, The Theory of Composites (Cambridge University Press, Cambridge, England, 2002).

[21] Z. Jacob, J. Y. Kim, G. V. Naik, A. Boltasseva, E. E. Narimanov, and V.M. Shalaev, Engineering Photonic Density of States Using Metamaterials, Appl. Phys. B 100, 215 (2010).

[22] S. Foteinopoulou, M. Kafesaki, E. N. Economou, and C. M. Soukoulis, Two-Dimensional Polaritonic Photonic Crystals as Terahertz Uniaxial Metamaterials, Phys. Rev. B 84, 035128 (2011).

[23] M. Schubert, T. E. Tiwald, and C. M. Herzinger, Infrared Dielectric Anisotropy and Phonon Modes of Sapphire, Phys. Rev. B 618187 (2000).
[24] W. S. Boyle, A. D. Brailsford, and J. K. Galt, Dielectric Anomalies and Cyclotron Absorption in the Infrared: Observations on Bismuth, Phys. Rev. 109, 1396 (1958).

[25] W. S. Boyle and A. D. Brailsford, Far Infrared Studies of Bismuth, Phys. Rev. 120, 1943 (1960).

[26] V. D. Kulakovskii and V. D. Egorov, Plasma Reflection in Bismuth and Bismuth-Antimony Alloys, Sov. Phys. Solid State 15, 1368 (1974).

[27] L. V. Alekseyev, V. A. Podolskiy, and E. E. Narimanov, Homogeneous Hyperbolic Systems for Terahertz and Far-Infrared Frequencies, Adv. OptoElectron. 2012, 267564 (2012).

[28] A. Hadni and X. Gerbaux, Far IR Excitation of Longitudinal Optical Phonons in Triglycine Sulphate, Ferroelectrics 248, 15 (2000).

[29] X. Gerbaux, M. Tazawa, and A. Hadni, Far IR Transmission Measurements on Triglycine Sulphate (TGS), at $5 \mathrm{~K}$, Ferroelectrics 215, 47 (1998).

[30] T. Dumelow, J. A. P. da Costa, and V. N. Freire, Slab Lenses from Simple Anisotropic Media, Phys. Rev. B 72, 235115 (2005).

[31] J. Sun, J. Zhou, B. Li, and F. Kang, Indefinite Permittivity and Negative Refraction in Natural Material: Graphite, Appl. Phys. Lett. 98, 101901 (2011).

[32] I. I. Smolyaninov and E. E. Narimanov, Metric Signature Transitions in Optical Metamaterials, Phys. Rev. Lett. 105, 067402 (2010).

[33] Z. Jacob, I. I. Smolyaninov, and E. E. Narimanov, Broadband Purcell Effect: Radiative Decay Engineering with Metamaterials, Appl. Phys. Lett. 100, 181105 (2012); see also arXiv:0910.3981v1.

[34] M. A. Noginov, H. Li, H. Yu, A. Barnakov, D. Dryden, G. Nataraj, G. Zhu, C. E. Bonner, M. Mayy, Z. Jacob, and E. E. Narimanov, Control of Spontaneous Emission with Metamaterials, Opt. Lett. 35, 1863 (2010).

[35] J. Kim, V. P. Drachev, Z. Jacob, G. V. Naik, A. Boltasseva, E. E. Narimanov, and V. M. Shalaev, Improving the Radiative Decay Rate for Dye Molecules with Hyperbolic Metamaterials, Opt. Express 20, 8100 (2012).

[36] E. E. Narimanov, H. Li, Yu. A. Barnakov, T. U. Tumkur, and M. A. Noginov, Reduced Reflection from Roughened Hyperbolic Metamaterial, Opt. Express 21, 14956 (2013).

[37] H. N. S. Krishnamoorthy, Z. Jacob, E. E. Narimanov, I. Kretzschmar, and V. M. Menon, Topological Transitions in Metamaterials, Science 336, 205 (2012).

[38] D. Lu, J. J. Kan, E. E. Fullerton, and Z. Liu, Enhancing Spontaneous Emission Rates of Molecules Using Nanopatterned Multilayer Hyperbolic Metamaterials, Nat. Nanotechnol. 9, 48 (2014).

[39] M. Born and E. Wolf, Principles of Optics, 7th (expanded) ed. (Cambridge University Press, Cambridge, England, 2013), p. 838 .

[40] N. W. Ashcroft and N. D. Mermin, Solid State Physics (Cengage Learning, Stamford, 1976).

[41] V. A. Podolskiy and E. E. Narimanov, Strongly Anisotropic Waveguide as a Nonmagnetic Left-Handed System, Phys. Rev. B 71, 201101(R) (2005).

[42] J. B. Pendry, Radiative Exchange of Heat between Nanostructures, J. Phys. Condens. Matter 11, 6621 (1999). 
[43] S.-A. Biehs, E. Rousseau, and J.-J. Greffet, Mesoscopic Description of Radiative Heat Transfer at the Nanoscale, Phys. Rev. Lett. 105, 234301 (2010).

[44] E. Rousseau, A. Siria, G. Jourdan, S. Volz, F. Comin, J. Chevrier, and J.-J. Greffet, Radiative heat transfer at the nanoscale, Nat. Photonics 3, 514 (2009).

[45] Y. Guo, C. L. Cortes, S. Molesky, and Z. Jacob, Broadband super-Planckian thermal emission from hyperbolic metamaterials, Appl. Phys. Lett. 101, 131106 (2012).

[46] S.-A. Biehs, M. Tschikin, and P. Ben-Abdallah, Hyperbolic Metamaterials as an Analog of a Blackbody in the Near Field, Phys. Rev. Lett. 109, 104301 (2012).

[47] J.-J. Greffet and C. Henkel, Coherent Thermal Radiation, Contemp. Phys. 48, 183 (2007).

[48] A. M. DaSilva, Y.-C. Chang, T. Norris, and A.H. MacDonald, Photonic Density of States Enhancement in Finite Graphene Multilayers, Phys. Rev. B 88, 195411 (2013)

[49] L. V. Alekseyev, V. A. Podolskiy, and E. E. Narimanov, Homogeneous Hyperbolic Systems for Terahertzand Far-Infrared Frequencies, Adv. OptoElectron. 2012, 267564 (2012).

[50] P. R. West, S. Ishii, G. V. Naik, N. K. Emani, V. M. Shalaev, and A. Boltasseva, Searching for Better Plasmonic Materials, Laser Photonics Rev. 4, 795 (2010).
[51] J. Elser, R. Wangberg, V. A. Podolskiy, and E. E. Narimanov, Nanowire Metamaterials with Extreme Optical Anisotropy, Appl. Phys. Lett. 89, 261102 (2006).

[52] M. A. Noginov, Y. A. Barnakov, G. Zhu, T. Tumkur, H. Li, and E. E. Narimanov, Bulk Photonic Metamaterial with Hyperbolic Dispersion, Appl. Phys. Lett. 94, 151105 (2009).

[53] J. Yao, Z. Liu, Y. Liu, Y. Wang, C. Sun, G. Bartal, A. M. Stacy, and X. Zhang, Optical Negative Refraction in Bulk Metamaterials of Nanowires, Science 321, 930 (2008).

[54] V. N. Smolyaninova, B. Yost, D. Lahneman, E. E. Narimanov, and I. I. Smolyaninov, Self-Assembled Tunable Photonic Hyper-crystals, arXiv:1312.7138.

[55] P. Yeh, Optical Waves in Layered Media (Wiley, Hoboken, New Jersey, 2005).

[56] S. Foteinopoulou, M. Kafesaki, E. N. Economou, and C. M. Soukoulis, Backward Surface Waves at Photonic Crystals, Phys. Rev. B 75, 245116 (2007).

[57] H. H. Li, Refractive Index of Silicon and Germanium and Its Wavelength and Temperature Derivatives, J. Phys. Chem. Ref. Data 9, 561 (1980).

[58] R. Buckley and P. Berini, Figures of Merit for 2D Surface Plasmon Waveguides and Application to Metal Stripes, Opt. Express 15, 12174 (2007). 